\title{
Andreas Roth Das Bild von Religion und Kirche bei ostdeutschen Tageszeitungsredakteuren
}

In den Tagen der SED-Diktatur war die Kommunikation zwischen Journalisten und Kirchenvertretern auf dem Gebiet der damaligen DDR ein vermintes Feld. Ein DDR-Redakteur war meist in Personalunion zugleich Vertreter des Herrschaftsapparates; Christen und Kirche wurden von ihm kritisiert oder doch zumindest ignoriert. Kirchenleute ihrerseits hielten aus diesen Gründen meist strenge Distanz zu den Medienvertretern. Die DDR ist mittlerweile Geschichte, was von ihr bleibt ist auch eine religionssoziologisch messbare Hinterlassenschaft: Die große Mehrheit der Ostdeutschen sind weder Mitglied einer Kirche, mehr noch: sie sind oft „religiös unmusikalisch" 1 . Was aber bedeutet dies für die Kommunikation zwischen Kirche und Medien in Ostdeutschland? Welche Eigenschaften und Lebensäußerungen von Kirche nehmen ostdeutsche Journalisten als berichtenswert wahr, welche nicht? Und wodurch wird deren religionsrelevantes journalistisches Verhalten bedingt, welche Einstellungen, Wahrnehmungsmuster und Werturteile sind dafür bestimmend?

$\mathrm{Zu}$ diesen Fragen fehlten bislang empirische Untersuchungen. Im deutschen Sprachraum steht bei der Erforschung von religiösen Einstellungen von Journalisten die Studie des österreichischen Kommunikationswissenschaftlers Maximilian Gottschlich $(1995)^{2}$ im Vordergrund. Aufgrund deren abweichender Fragestellung und Methodik, vor allem aber angesichts der gänzlich anderen religionssoziologischen Situation in Österreich lässt sich Gottschlichs Studie für die explorative Untersuchung der ostdeutsche Situation jedoch nicht als Referenz heranziehen. Die vorliegende Studie wurde im Januar 2004 unter der Betreuung von Prof. Dr. Wolfgang Ratzmann am Institut für Praktische Theologie der Universität Leipzig als Examensarbeit eingereicht und verteidigt.

1 Vgl. hierzu Detlef Pollack: Der Wandel der religiös-kirchlichen Lage in Ostdeutschland nach 1989. In: Detlef Pollack/ Gerd Pickel (Hg.): Religiöser und kirchlicher Wandel in Ostdeutschland 1989-1999. Opladen 2000, S. 18-47.

2 Maximilian Gottschlich: Was „glauben“ Österreichs Journalisten? Untersuchung über die religiöse Einstellung und Wertorientierung der Medien-Elite. In: Communicatio Socialis, 3/1995, S. 303-320. Ebenfalls österreichbezogen ist die Dissertation von Gabriele Russ: Die Kirche der Journalisten. Graz 2002. 


\section{Untersuchungsplan und Untersuchungsmethoden}

Als Feld für die empirische Untersuchung der Kommunikation zwischen Kirche und medialer Öffentlichkeit in Ostdeutschland wurden die beiden Regionalblätter Sächsische Zeitung und Leipziger Volkszeitung samt ihren Lokalausgaben gewählt. Gründe hierfür waren einerseits die hohe Bedeutung von Lokalzeitungen für die Information über Religion und Kirche $^{3}$, andererseits die ostdeutsche Zeitungsstruktur, die von wenigen auflagenstarken Regionaltiteln geprägt ist. ${ }^{4}$ Befragt werden sollten all jene Redakteure dieser Zeitungen, in deren Aufgabengebiet die Beschäftigung mit Kirche und Religion in Sachsen fallen würde: Lokalredakteure und Redakteure der für das politische Zeitgeschehen zuständigen Ressorts. ${ }^{5}$

Von vornherein wurde wegen des engen zeitlichen und finanziellen Rahmens dieser Untersuchung als Methode für die Datenerhebung der Einsatz eines standardisierten Fragebogens favorisiert. Wegen dieser Rahmenbedingungen mussten weitere methodische Einschränkungen in Kauf genommen werden: 1. Die Nutzung von Daten- und Fragenarchiven, um die neu zu gewinnenden Ergebnisse in eine Trendreihe einzubetten, war aufgrund der Forschungssituation kaum möglich. 2. Pre-Tests der Fragen und Erhebungsprozeduren unter Feldbedingungen mussten durch einen Testlauf mit nicht der Zielgruppe zugehörigen Versuchspersonen ersetzt werden, die den Fragebogen auf seine Verständlichkeit hin prüften. 3. Auf eine Prüfung der Stabilität des Meinungsbildes über einen Zeitraum (Re-Tests) musste ebenfalls verzichtet werden.

3 In der EKD-Studie von Klaus Engelhardt/ Hermann von Loewenich/ Peter Steinacker (Hg.): Fremde Heimat Kirche. Die dritte EKD-Erhebung über Kirchenmitgliedschaft. Gütersloh 1997, S. 417 wird die Bedeutung der Lokalzeitungen als sowohl für Kirchenmitglieder als für Konfessionslose mit Abstand wichtigstes Medium zur Information über kirchliches Geschehen hervorgehoben. Zur besonderen, oft monopolartigen Stellung der ehemaligen SED-Regionalzeitungen und ihrer hohen Bedeutung für Ostdeutschland vgl. Beate Schneider/ Klaus Schönbach/ Dieter Stürzebecher: Journalisten im vereinigten Deutschland. Strukturen, Arbeitsweisen und Einstellungen im Ost-West-Vergleich. In: Publizistik 3/1993, S. 353-382, $379 \mathrm{f}$.

4 Die Tageszeitungslandschaft in Sachsen wird in jedem Regierungsbezirk fast monopolartig beherrscht von je einer Regionalzeitung: der "Sächsischen Zeitung“, der "Leipziger Volkszeitung“ bzw. ihrer Tochterausgaben sowie der Chemnitzer "Freien Presse“. Hinzu kommen Boulevardzeitungen sowie im Vogtland, in Dresden und in Döbeln unabhängige, von ihrer Auflage her aber eher kleine Lokalzeitungen.

5 Es wurden an 18 Lokalredaktionen der "Sächsischen Zeitung" sowie an 8 der „Leipziger Volkszeitung“ je 5 Fragebögen verschickt, da diese Redaktionen jeweils 5-6 Redakteure beschäftigen (an die Dresdner und Leipziger Stadtredaktionen wurden 6 Bögen verschickt). An die Zentralredaktionen beider Zeitungen wurden je 9 Bögen versandt. 
Die vorliegende Untersuchung verfolgt das Ziel einer explorativen Analyse. Die Auswertung konzentriert sich zumeist aufgrund der geringen Stichprobe auf die deskriptive Zusammenfassung der Daten.

\section{Stichprobenbeschreibung}

Von den 150 versandten Fragebögen wurden 29 ausgefüllt zurückgeschickt, die Rücklaufquote beträgt damit 19,3\%. Von 18 an die Redakteure der beiden Zentralredaktionen von Leipziger Volkszeitung und Sächsischer Zeitung verschickten Bögen kamen 6 zurück (Rücklauf 33,3\%), von 132 an Lokalredaktionen der beiden Zeitungen verschickten Bögen waren es 23 (Rücklauf 17,4\%). Betrachtet man die Rücklaufquote bei vergleichbaren schriftlichen Befragungen und stellt man bei der vorliegenden Untersuchung eine gewisse Brisanz des Themas in Rechnung, so ist dieses Ergebnis durchaus noch als zufriedenstellend zu werten. Für eine erste Untersuchung des Themas stellt es aus der Sicht des Autors eine zuverlässige Datenbasis dar.

Befragungspersonen, die „sozial erwünscht“ oder „politisch korrekt“ antworten, wie auch eine aus unterschiedlichsten Gründen hohe Nonresponse-Rate, können die Daten systematisch verzerren (Bias). Angesichts der zu vermutenden Distanz bzw. Indifferenz vieler ostdeutscher Journalisten gegenüber dem Thema Kirche und Religion muss dies auch bei der Interpretation der vorliegenden Daten beachtet werden. Gerade wegen des unvollständigen Rücklaufs bleibt das Problem der Bewertung dieser Daten. Ein Vergleich der demographischen Werte der Stichprobe mit einer externen Verteilung kann hier nicht vorgenommen werden, da keine demographischen Daten zu einer Grundgesamtheit „Tageszeitungsredakteure in Ostdeutschland" vorliegen. Insofern kann diese Stichprobe nicht als repräsentativ für die Grundgesamtheit aller Tageszeitungsredakteure in 0stdeutschland angesehen werden; die Interpretation der vorliegenden Daten bezüglich jener Grundgesamtheit muss folglich immer auf hypothetischem Niveau bleiben.

Innerhalb der Rücklauf-Stichprobe wurden 15 Frauen (51,7\%) und 12 Männer $(41,4 \%)$ befragt, 2 machten zum Geschlecht wie auch zur Dauer ihrer Berufstätigkeit und ihrem Alter keine Angaben, wahrscheinlich, um ihre Anonymität zu wahren. Die Streuung des Alters der Befragungspersonen reicht von 28 bis 58 Jahre (Durchschnittsalter: 41 Jahre). Die befragten Journalisten sind im Schnitt fast 16 Jahre in ihrem Beruf tätig. $51,9 \%$ von ihnèn begannen ihre journalistische Arbeit ab 1990, 48,1\% waren bereits vor 1990 Journalisten. Da im Fragebogen auf die Frage nach der beruflichen Laufbahn in der DDR bewusst verzichtet wurde, um 
nicht den Verdacht einer Examinierung von Ex-DDR-Journalisten aufkommen zu lassen und damit die Beantwortung zu hemmen, kann zumindest dieses Ergebnis als ein Indiz für die berufliche Prägung der Journalisten durch die Vor- bzw. Nachwende-Gesellschaft angesehen werden. ${ }^{6}$

Die befragten Journalisten sind mehrheitlich nicht Mitglieder einer christlichen Kirche (79,3\%). Dieses Ergebnis spiegelt zum einen die Verhältnisse in der ostdeutschen Gesellschaft insgesamt wider und kann damit bezüglich der Religionszugehörigkeit als repräsentativ angesehen werden. Andererseits ist zu vermuten, dass bei Kirchenmitgliedern die Bereitschaft zum Ausfüllen des Fragebogens tendenziell höher sein könnte als bei Nicht-Mitgliedern, so dass der Anteil der Nicht-Kirchenmitglieder unter den ostdeutschen Journalisten möglicherweise insgesamt eher höher sein wird als in der Stichprobe dieser Studie. Unter den bereits vor 1990 tätigen Redakteuren in der Stichprobe war nur ein Kirchenmitglied, während immerhin 35,7\% der nach 1990 in den Journalismus gekommenen Redakteure Kirchenmitglieder sind.

\section{Was „glauben" ostdeutsche Journalisten?}

\subsection{Primäre Verstehens- und Erwartungsmuster der befragten Redakteure $z u$,Religion"}

Um das wichtigste Verstehensmuster von „Religion“ sowie die diesbezügliche Haupterwartung und das theoretische Einstellungskonzept der

6 Zur personellen Zusammensetzung der ostdeutschen Tageszeitungsredaktionen erklärt die Studie von Schneider u.a. (S. 355-357): Von den Tageszeitungsredakteuren in Ostdeutschland, die 1993 bei den ehemaligen SED-Bezirkszeitungen beschäftigt waren, sind $71 \%$ bereits vor 1989 journalistisch tätig gewesen, 20\% waren es ab 1990 und 9\% kamen aus Westdeutschland hinzu. Dieter Stürzebecher: Woher kommen sie, wie denken sie, was wollen sie? In: Michael Haller/Klaus Puder/Jochen Schlevoigt (Hrsg.): Presse Ost - Presse West. Journalismus im vereinten Deutschland. Berlin 1995, S. 207-225, 208 weist darauf hin, dass zwischen 1989 und 1995 38\% der festangestellten DDR-Redakteure ausgeschieden sind. Bei der „Leipziger Volkszeitung“ waren 1995 ca. zwei Drittel der Redakteure aus SED-Zeiten ausgeschieden, neu hinzugekommen sind Redakteure der DDR-Blockparteienpresse, der Nachwende-Presse sowie Westdeutsche (so Hartwig Hochstein: Die „Leipziger Volkszeitung“ zwischen Kontinuität und Wandel. In: Reinhard Bohse: Meinungsfreiheit - Medienfreiheit - Zeitenwende - Wendezeiten. Leipziger Rundtischgespräch zum Internationalen Tag der Pressefreiheit am 3. Mai 1995. Leipzig 1995, S. 53). Armin Scholl: Rollenverständnis ost- und westdeutscher Journalisten, in: Haller u.a., S. 226-231, 229 weist zu Recht darauf hin, dass bei der Bewertung der Herkunft der Redakteure im Blick sein muss, dass auch die Jahre nach 1989 sozialisierend gewirkt haben. 
Befragungsperson $\mathrm{zu}$ „Religion“ zu erfragen, sollte sie eines aus neun Items auswählen, mit dem sie den Satz „Die wichtigste Aufgabe einer Religion ist?"fortführen konnte.

Lässt man die einzige ungültig ausgefüllte Antwort hierzu unberücksichtigt, so ergibt sich folgendes Bild: Der mit Abstand größte Anteil der befragten Redakteure $(35,5 \%)$ sieht im Geben von ,moralischer Orientierung“, also in einer immanenten gesellschaftlichen Funktion, die vorrangige Aufgabe von Religion. Mit einigem Abstand folgen drei gleich große Gruppen (jeweils 17,9\%). Zwei dieser drei Gruppen antworten mit „transzendent" zu nennenden Items: Die einen sehen die erste Aufgabe der Religion in der „Vermittlung einer Beziehung zu Gott“, die zweite Gruppe sieht diese in der Hilfe, „einen Sinn im Leben zu finden“. Die mit ebenfalls 17,9\% gleich große dritte Gruppe sieht die vorrangige Aufgabe von Religion ganz immanent in der „Weitergabe von Tradition und Kultur“. 7,1\% der Befragten sehen die Aufgabe der Religion möglicherweise als Nachhall der philosophischen Religionskritik des 19. Jahrhunderts und der kommunistischen Ideologie als „die Verbreitung einer Weltanschauung, die nicht mehr in die moderne Welt passt" (unter den schon vor 1990 Tätigen: 16,7\%). Nur 3,6\% sehen darin, „Menschen über Leid und Ungerechtigkeit zu trösten", eine primäre Aufgabe von Religion. Gar keine Zustimmung fanden die klassisch religionskritischen Items, die erste Aufgabe einer Religion sei „die gesellschaftliche Ordnung zu bewahren" oder „ein Mittel zur Stabilisienung von Herrschaft“. Ebenfalls keine Zustimmung fand das transzendente Item, Religion sei zuerst dazu da, um ein Leben nach dem Tod zu erlangen.

Unter den bereits vor 1990 tätigen Redakteuren finden die Items zur moralischen und kulturellen Funktion von Religion überdurchschnittlich hohe Zustimmung; bei den seit 1990 Tätigen sind es die Items zur Gottesbeziehung und Sinnfindung.

\subsection{Verstehens-, Einstellungs- und Erwartungsmuster zu „Kirche“}

In diesem Komplex hatten die Befragungspersonen die Möglichkeit, den Satz „Die Kirche ist für mich?“ mit Hilfe von 19 Ergänzungen fortzusetzen, wobei hier die Auswahl mehrerer Items möglich war. Damit sollte ein differenziertes persönliches Einstellungskonzept bezüglich „Kirche“ erfragt werden. Die Anteile der Befragten, die eine einschlägige Antwort wählten, schwanken zwischen null und fast 38 Prozent.

Die meiste Zustimmung unter den Befragten erhielt mit 37, 9\% die Antwort, Kirche sei „eine in Ostdeutschland eher unbedeutende Organisation“. Dies kann als nüchterne Feststellung gewertet werden, aber auch als Hinweis auf eine grundsätzlich indifferente Haltung gegenüber 
dem Phänomen „Kirche“. Nimmt man die Ergebnisse zu Verbundenheit und Vertrautheit mit Kirche hinzu (s. Kapitel 3.3 und 3.5), so wird man hierbei jedoch von Indifferenz ausgehen dürfen. Jedoch muss das Ausmaß der Indifferenz eingeschränkt werden: Trotz des hohen Anteils von Nichtkirchenmitgliedern und Kirchenfernen unter ihnen geben doch nur $13,8 \%$ der Befragten an: „Kirche hat für mich gar keine Bedeutung“. Gar nur 10,3\% sehen in Kirche einen „Verein unter vielen“, und nur 6,9\% „eine Interessengruppe wie z.B. die Gewerkschaften“.

Eine solche eingeschränkt indifferente Einstellung der Befragten mit einer Tendenz zur skeptischen Distanz bildet einen Hauptstrom des sich in dieser Frage ergebenden Bildes. 27,6\% der antwortenden Redakteure geben an, Kirche sei „eine Organisation, der ich skeptisch gegenüberstehe“. Und $20,7 \%$ sehen ebenfalls distanziert und skeptisch in der Kirche eine „hierarchisch organisierte Machtinstitution“. Jedoch ist auch hier zu beobachten, dass die skeptische Haltung gegenüber Kirche und Religion meist ohne die polemischen Kategorien der klassischen und auch realsozialistischen Religionskritik auskommt. Am ehesten wird Kirche noch als "überholte Weltanschauung“ angesehen (13,8\% Zustimmung; bei den bereits vor 1990 Tätigen: 23,1\%). Die von der Religionskritik vertretene Auffassung, Kirche sei Instrument der Herrschenden oder zur Herrschaftsstabilisierung, findet erstaunlicherweise unter den von ihrer Profession her herrschaftskritischen Journalisten sehr wenig Zustimmung. Nur 6,9\% sehen in der Kirche "eine Organisation, die der CDU näher steht als anderen Parteien" oder „eine Organisation zur Erhaltung des derzeitigen Gesellschaftssystems“. Nicht überraschend dafür ist, dass eine von einem christlichen Boden aus erhobene Kirchenkritik in einer als areligiös $z \mathfrak{u}$ bezeichnenden ostdeutschen Gesellschaft so nicht vertreten wird. Kirche sei ,eine hierarchische Institution, die den Geist Jesu Christi erstickt" findet nur bei einem Befragten Zustimmung.

Neben diesem großen Anteil an indifferent bis skeptisch-distanzierten Einstellungen gibt es eine fast genauso breite Strömung von freundlichpositiven Einstellungen gegenüber Kirche, ohne dass dies automatisch als Übereinstimmung oder große Nähe gewertet werden darf. Eher wird man hier in der Zusammenschau mit den Angaben zur Kirchenmitgliedschaft, -vertrautheit und -verbundenheit von einer Anerkennung aus einer gewissen Distanz sprechen dürfen. In Analogie zu den Antworten auf die Frage nach der primären Aufgabe von Religion (s. Kapitel 3.1) sind 34,5\% der Antwortenden der Ansicht, Kirche sei „eine Institution zur moralischen Orientierung in der Gesellschaft" sowie „wichtig für unsere Kultur“. Ebenfalls oft wurde mit jeweils $31 \%$ die Antwort, Kirche sei "eine Organisation, die sich um die Armen und Schwachen kümmert" 
ausgewählt, womit das positive Image von Kirche als sozial engagierte Institution offenbar wird. 17,2\% schätzen Kirche als „eine kritische Stimme in der Politik" - mehr als der Anteil derjenigen, die Kirche in einer großen Herrschaftsnähe sehen.

Bei einem großen Teil der Antwortenden ist auch die religiöse Dimension von Kirche im Blick, jedoch aus einer Distanz zur eigenen Betroffenheit: Wenn 31\% der Antwortenden in Kirche „eine Hilfe zum Finden von Sinn im Leben" sehen, ist dies zwar die häufigste im engeren Sinne "religiöse“ Antwort, inhaltlich bleibt sie jedoch unscharf und nicht spezifisch christlich. Ähnlich ist es zu bewerten, wenn ein Fünftel der Antwortenden $(20,7 \%)$ die Kirche als ,eine Möglichkeit, eine Beziehung zu Gott aufzubauen", bezeichnen. Immerhin mehr als ein Viertel der Antwortenden $(27,6 \%)$ jedoch wählten auch die spezifisch christliche Antwort, Kirche sei „die Gemeinschaft derjenigen, die Jesu Christi nachfolgen wollen".

Während all diese Antworten auch aus einer Distanz zur eigenen Existenz gegeben werden konnten, erhielten jene Items, die eine persönliche Verbundenheit mit Kirche implizierten, sehr geringe Zustimmungsraten. Nur 6,9\% halten Kirche für einen "tragenden Grund meines Lebens“. Und wie in der Religionsfrage wählte auch hier keiner, dass Kirche für ihn „eine Hoffnung auf ein Weiterleben nach dem Tod" sei. Dieser Befund korrespondiert mit dem Bild zur Religiosität und Kirchenverbundenheit der befragten Redakteure.

Bemerkenswert ist, dass die erst ab 1990 tätigen Befragten die Kirche in ihrer politischen Dimension kritischer betrachten als ihre schon länger arbeitenden Kollegen. ${ }^{7}$ Auch betonen sie die religiösen Dimensionen von „Kirche“ wesentlich stärker, während ihre schon vor 1990 tätigen Kollegen eher die kulturelle Bedeutung von Kirche betonen.

\subsection{Vertrautheit mit dem christlichen Gemeindeleben}

Den auf dem Fragebogen vorgegebenen Satz „Christliches Gemeindeleben ist mir vertraut" konnten die Befragungspersonen mit dem Ankreuzen einer Ziffer auf einer Skala zwischen 0 („überhaupt nicht“) und 5 („sehr vertraut") vervollständigen.

Das Ergebnis zeigt ein Gefälle: Demnach bezeichnen sich 27,6\% der Befragten als „überhaupt nicht“ vertraut mit dem Gemeindeleben, $41,7 \%$

$723,1 \%$ der bereits vor 1990 tätigen Redakteure in der Stichprobe schätzen Kirche als „kritische Stimme in der Politik“, mehr als unter den nach 1990 in den Beruf gekommênen. Letztere sehen häufiger in der Kirche eine Machtinstitution (35,7\% Zustimmung) und eine große CDU-Nähe (14,3\% Zustimmung). 
sind wenig bis etwas vertraut, $27,6 \%$ stärker vertraut und nur $3,4 \%$ "sehr vertraut" mit dem christlichen Gemeindeleben. In diesem Ergebnis darf man möglicherweise auch ein enges Verhältnis zum Maß des Wissens über Kirche und kirchliches Leben vermuten.

\section{Abbildung 1: Selbsteinschätzung der Vertrautheit mit Kirche}

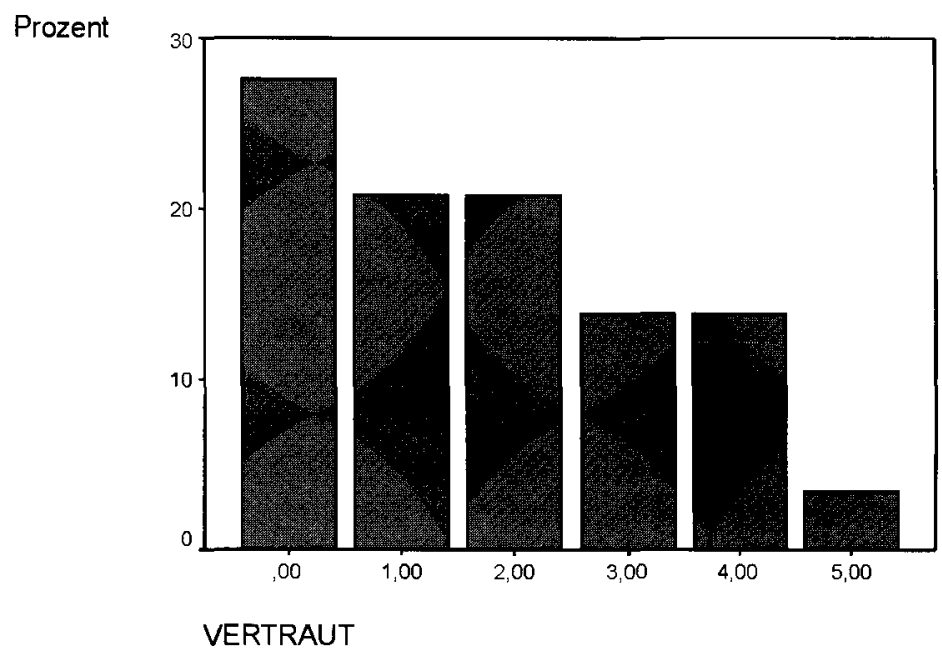

\subsection{Selbsteinschätzung der eigenen Religiosität}

Zur Vervollständigung des Satzes „Ich würde mich als religiös bezeichnen“ stand ebenfalls eine Skala von 0 („überhaupt nicht“) bis 5 („unbedingt“) zur Auswahl.

$62,1 \%$ der Befragten gaben an, sich „überhaupt nicht“ als religiös bezeichnen zu können (unter den bereits vor 1990 Tätigen: 69,2\%; unter den ab 1990 Tätigen: 50,0\%8), 13,8\% sehen sich als kaum religiös, $20,6 \%$ als mittelmäßig bis stärker religiös und nur ein Befragter $(3,4 \%)$ würde sich als „unbedingt“ religiös bezeichnen.

8 In der Gesamtbevölkerung Ostdeutschlands liegt der Wert bei $39 \%$, so die neue Studie von Johannes Christian Koecke/ Matthias Sachs: Religion - Politik - Gesellschaft. Ergebnisse einer repräsentativen Umfrage. Arbeitspapier/Dokumentation der Konrad-Adenauer-Stifung Nr. 110, Sankt Augustin 2003, S. 3, 21. 19\% der 0stdeutschen würden sich demnach als "ziemlich religiös“ einschätzen. Bei einem Vergleich ist jedoch der Unterschied in der Erhebungsmethode und die NichtRepräsentativität der vorliegenden Erhebung zu beachten. 
Abbildung 2: Selbsteinschätzung der eigenen Religiosität

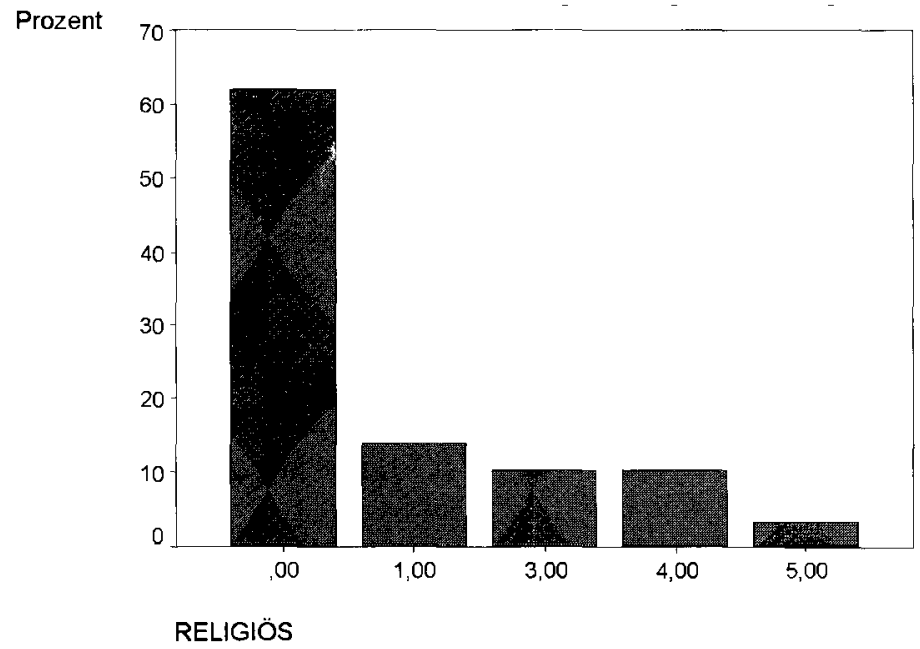

\subsection{Verbundenheit mit der Kirche}

Auch der Satz "Ich fühle mich mit der Kirche verbunden "konnte auf einer Skala zwischen 0 („überhaupt nicht“) und 5 („sehr verbunden“) eingeordnet werden.

$44,8 \%$ der Befragten fühlen sich „überhaupt nicht“ (unter den bereits vor 1990 Tätigen: $61,5 \%$; bei den ab 1990 Tätigen: $28,6 \%$ ), 20,7\% kaum bzw. wenig mit der Kirche verbunden. 31,0\% fühlen sich mittelmäßig bis stärker und nur 3,4\% (ein Befragter) "sehr verbunden“ mit der Kirche. ${ }^{9}$

Abbildung 3: Kirchenverbundenheit

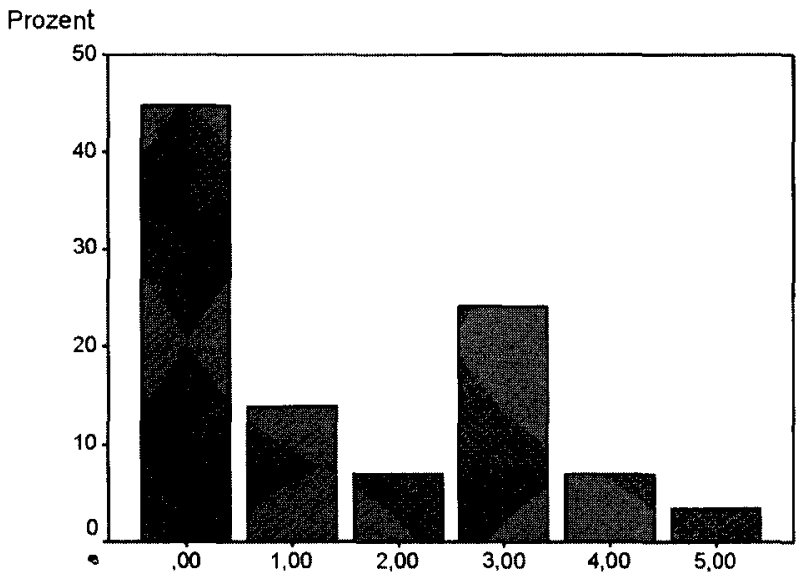

9 Vgl. dazu die Werte von Koecke/Sachs, S. 7: 11\% der Ostdeutschen fühlten sich "sehr stark bis stark" mit der Kirche verbunden, 48\% „überhaupt nicht". 
3.6 Die Einschätzung des Verhältnisses der Berufskollegen zu Religion und Kirche

Zur Vervollständigung des Satzes „Das Verhältnis meiner journalistischen Berufskollegen zu Religion und Kirche würde ich bezeichnen als?" standen vier Antwortoptionen zur Auswahl.

Lässt man die beiden ungültigen Antworten unberücksichtigt, so bezeichneten mit 40,7\% die meisten Befragten die Einstellung ihrer Berufskollegen zu Religion und Kirche als „neutral-gleichgültig“. Fast ebenso viele Befragte $(37,0 \%)$ bezeichneten dieses Verhältnis der Journalisten als „freundlich-positiv“. Nur 18,5\% werteten es als "distanziertkritisch“ und nur 3,7\% (ein Befragter) als „ablehnend-feindlich“.

Abbildung 4: Einstellung der Kollegen gegenüber Religion und Kirche

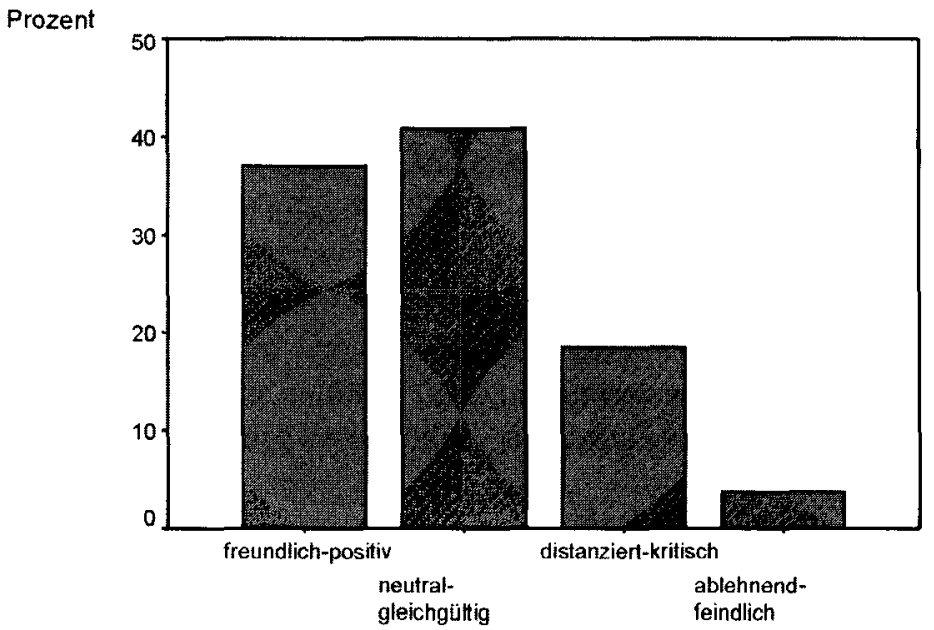

\section{Die Einschätzung der journalistischen Relevanz kirchlicher Themen}

Bei der Frage nach den Faktoren und Eigenschaften des kirchlichen Lebens, die es im journalistischen Auswahlprozess interessant machen, musste mangels vergleichbarer Studien eine eigene Methode entwickelt werden. Zentral war dabei die Überlegung, dass sich der journalistische Auswahlprozess am genauesten anhand der journalistischen Reaktion auf konkret ausformulierte Themen messen lässt. Deshalb wurden z.T. auf Basis echter kirchlicher Pressemitteilungen 22 (für Lokalredakteure) bzw. 25 (für Redakteure der Zentralredaktionen) Kirchen-Themen ent- 
wickelt, die in einer überschriftartigen Zeile formuliert wurden und deren Priorität bei normaler Nachrichtenlage von den Befragten auf einer sechsstufigen Skala eingeschätzt werden sollte. ${ }^{10}$

Grundsätzlich wurde von den Befragungspersonen die gesamte vorgegebene Spannweite von 0 (,geringe Priorität“, d.h. das Thema wird nicht aufgegriffen) bis zu 5 ("hohe Priorität“, d.h. das Thema wird in einem größeren Beitrag verarbeitet) ausgeschöpft. Beim Vergleich der Mittelwerte der den verschiedenen Themen zuerkannten Prioritäten jedoch zeigt sich, dass der Durchschnitt der befragten Redakteure über alle der angegebenen Themen zumindest eine kleine Meldung, mitunter gar längere Beiträge veröffentlicht hätte. Kirchen-Themen sind für sie also relevant, obwohl sie selbst wie ihre Leserschaft mehrheitlich (70-80\%) kirchenfern sind.

Aufschlussreich ist das breite Spektrum der so eingeschätzten Kirchen-Themen: Nicht nur Themen, die Kirche in ihren sozialen und kulturellen Dimensionen zeigen, erhielten eine erhöhte Aufmerksamkeit der Befragten. Auch kirchliche Ereignisse und Prozesse mit theologischen und spirituellen Implikationen wie z.B. das Gelingen oder Misslingen von Ökumene auf lokaler Ebene wurden von den befragten Journalisten durchschnittlich mit mindestens mittlerer Priorität bedacht. Und nicht zuletzt waren es kirchliche Äußerungen, die mit dem Mut zum Konflikt Kritik an sozialen, wirtschaftlichen und politischen Zuständen übten, die eine hohe Beachtung bei den befragten Redakteuren fanden.

Dieses Ergebnis kann einerseits für eine grundsätzliche Aufgeschlossenheit der befragten Journalisten gegenüber kirchlichen Themen sprechen. Andererseits kann hier jedoch auch aus methodischen Gründen eine Verzerrung vorliegen. Zum einen kann schon bei der Konstruktion der Themen zu sehr der Blick auf das ,journalistisch Interessante" leitend gewesen sein. Zudem ist mit einem Fragebogen eine Redaktionssituation mit ihren verschiedenen Faktoren (z.B. die Informationsquelle, der Zeit- und Platzdruck etc.) nicht komplett konstruierbar. Ein genaueres Bild der redaktionellen Prioritäten könnte eine kommunikationswissenschaftliche Inhaltsanalyse der jeweiligen Medien geben.

10 Der methodische Nachteil bei diesem Verfahren ist, dass eine letztlich klare, disjunkte Abgrenzung und Gleichwertigkeit der inhaltlichen Faktoren und Themen nicht angenommen werden kann. Der - m.E. dem Thema angemessene und darum überwiegende - Vorteil dieses Vorgehens ist jedoch, dass es eine hohe Nähe zur journalistischen Praxis bedeutet, da dort die Themen und ihre Faktoren genau wie in dieser Untersuchung nur in verschiedenartigsten Mischformen vorliegen. Hätte man nur absţrakt nach den Prioritäten verschiedener inhaltlicher Faktoren gefragt, bestünde die Gefahr, dass das Ergebnis durch "sozial erwünschtes“ Antworten stärker verzerrt werden würde. 


\section{Die Kommunikation zwischen den befragten Redakteuren und der Kirche}

\section{1 Häufigkeit der Nutzung von Informationsquellen über Kirche und christliches Leben}

Die an alle befragten Redakteure gerichtete Leitfrage dieses Fragekomplexes lautete: "Woher erhalten Sie Ihre Informationen über Kirche und christliches Leben?" In der Frageanleitung wurde diese Fragestellung auf die evangelische Kirche in Sachsen hin konkretisiert.11 Die Befragten sollten auf einer sechsstufigen Skala (von $0 /$ nie bis $5 /$ sehr oft) die Häufigkeit der Nutzung einer Quelle einschätzen.

Insgesamt liegt die eingeschätzte Häufigkeit der beruflichen Kirchenkontakte der befragten Redakteure auf einem statistisch niedrigem $\mathrm{Ni}$ veau: Nur zwei Informationsquellen über Kirche und Religion werden „regelmäßig“" genutzt, alle anderen „selten“, der größte Teil „sehr selten“ bis "nie“.

\subsubsection{Die Informationsquellen der Lokalredakteure}

Die wichtigste Informationsquelle für die befragten Lokalredakteure ist der persönliche Kontakt zu Pfarrern; er wird „selten“, jedoch mit einer leichten Tendenz hin zur Regelmäßigkeit genutzt.

Im Gesamtbild (Abbildung 5) lassen sich signifikante Unterschiede zwischen den Mittelwerten der relativ hoch eingeschätzten Nutzungshäufigkeit der Quellen „Pfarrer“, „Pressemitteilungen der Gemeinden“, „Mitarbeiter der Gemeinden“ und teilweise auch „engagierte Gemeindemitglieder" einerseits und den extrem niedrig eingestuften Quellen „Kirchenzeitung Der Sonntag“, „Internetpräsenz der Superintendentur bzw. des Kirchenbezirkes“ und „persönlicher Kontakt zum Landeskirchenamt" feststellen.

Persönliche Kontakte vor Ort haben also erwartungsgemäß im eng umgrenzten Umfeld einer Lokalredaktion einen höheren Stellenwert als die Medienarbeit der räumlich entfernten Landeskirche. Betrachtet man die Rangordnung der eingeschätzten Häufigkeiten, so kann diese als Hinweis auf Schwachpunkte gelesen werden: Gerade im Internet - das mittlerweile $\mathrm{zu}$ den wichtigsten Recherchequellen für Journalisten gehört - können die Redakteure offenbar kaum aktuelle und für sie relevante Informationen über die Kirche vor Ort (Gemeinden und Superinten-

11 Ein Manko dieser Fragen ist, dass sie nicht explizit auf die Strukturen der Niederschlesischen Kirche bzw. der Kirchenprovinz Sachsen zugeschnitten sind, die sich in den Landkreisen Nord- und Ostsachsen z.T. mit den Zuständigkeitsbereichen einiger befragter Redaktionen überschneiden. 
denten) finden. Eine zweite Beobachtung bietet einen Hinweis darauf, dass der Superintendent, seine Mitarbeiter und Angebote - die ja oft strukturell ein ähnliches Gebiet betreuen wie eine Lokalredaktion kaum als Informationsquelle wahrgenommen werden. Dies könnte ein Hinweis sein auf die geringe Kenntnis der kirchlichen Strukturen seitens der Redakteure, aber möglicherweise auch auf die fehlende Wahrnehmung der Aufgabe der Öffentlichkeitsarbeit seitens der mittleren Kirchenebene.

\section{Abbildung 5: Die Informationsquellen der Lokalredakteure}

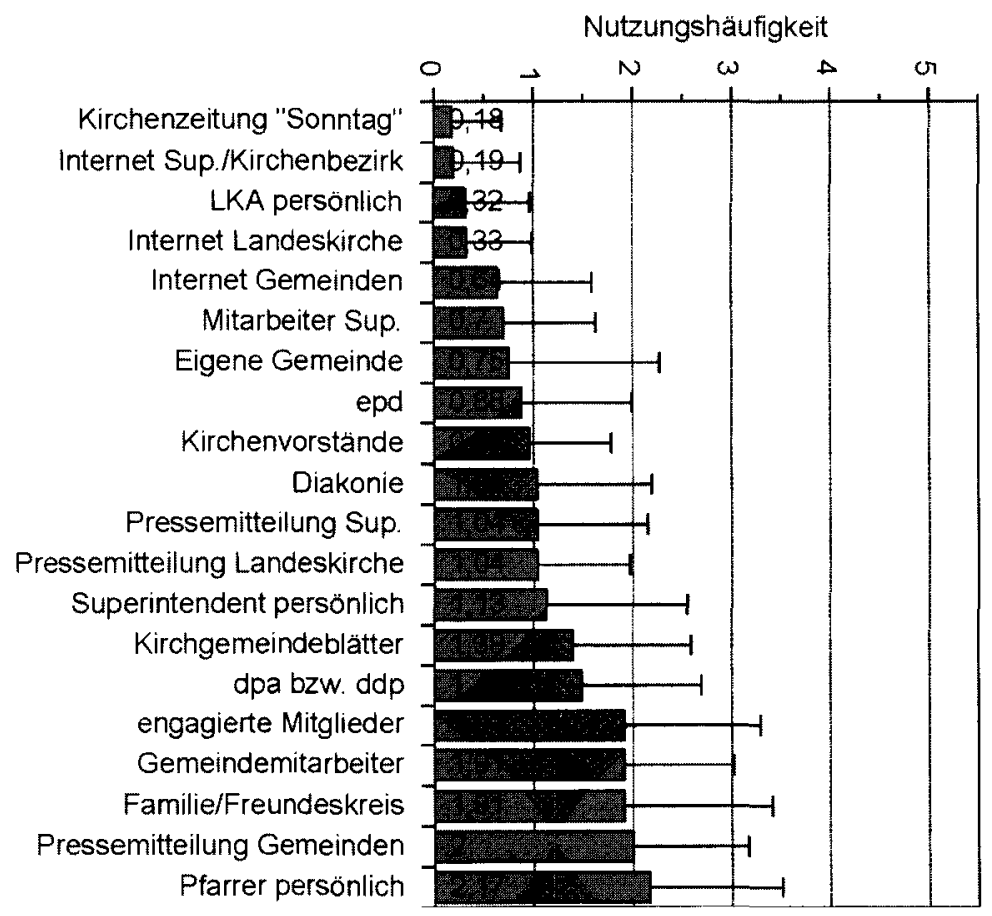

\subsubsection{Die Informationsquellen der Zentralredakteure}

Im Gegensatz zu den Lokalredakteuren erhalten die befragten Redakteure der Zentralredaktionen ihre Informationen über Kirche vorrangig nicht aus persönlichen Kontakten, sondern aus den schriftlichen Quellen der Nachrichtensysteme (Abbildung 6). „Regelmäßig“ und damit als wichtigste Quelle über kirchliche Themen nutzen sie die Nachrichtenagenturen epd und dpa bzw. ddp. „Selten“, jedoch mit einer Tendenz zur Regelmäßigkeit werden auch die Pressemitteilungen der evangelischen Landeskirche genutzt. Alle anderen Informationsquellen folgen - gemessen an der Rangordnung - mit großem Abstand und werden "sehr selten" bis "nie“ von den Redakteuren genutzt. Da hierfür jedoch nur 
Abbildung 6: Die Informationsquellen der Zentralredakteure

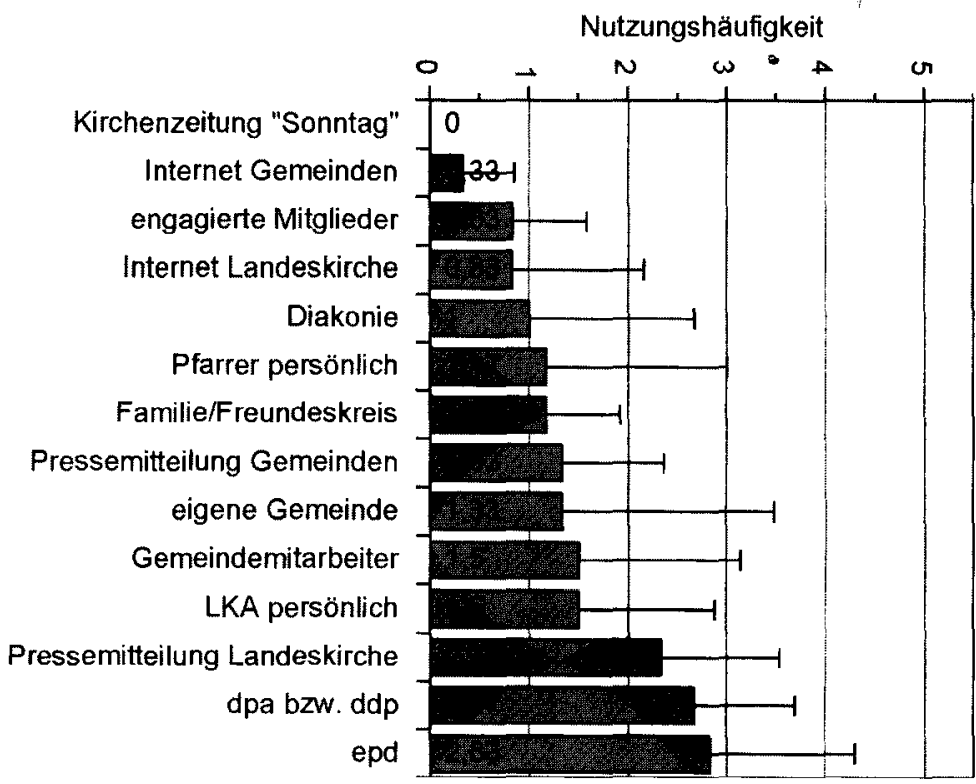

eine geringe Zahl von Redakteuren der Zentralredaktionen befragt werden konnte, war eine mathematische Prüfung auf signifikante Unterschiede zwischen den eingeschätzten Häufigkeitsangaben nicht möglich bzw. sinnvoll.

\subsection{Die Qualität der genutzten Informationsquellen über Kirche und christliches Leben}

$\mathrm{Zu}$ jeder auf dem Fragebogen aufgeführten Informationsquelle über Kirche und christliches Leben wurde zusätzlich die Frage gestellt: „Wie oft fließen diese Informationen in Ihre Artikel ein?". Die Frage nach der Einschätzung dieser Häufigkeit sollte hier als ein Kriterium für die Qualität der Quellen dienen, denn es ist anzunehmen, dass sie die Verwertbarkeit und den Gehalt der aus der Quelle gewonnenen Informatio. nen widerspiegelt.

Das Ergebnis in Kürze: Auch wenn die befragten Redakteure aus einer kirchlichen Quelle Informationen bekamen, setzen sie diese nur "selten“ in einen Artikel um. Die größte eingeschätzte Häufigkeit bei der Umsetzung erzielten nach der Rangskala Informationen, die aus der eigenen Gemeinde, aus den persönlichen Kontakten zu Pfarrern und Superintendenten, also allesamt aus persönlichen Begegnungen gewonnen werden: Sie sind „regelmäßig“ in Artikel umsetzbar. 


\subsection{Die Häufigkeit der Bearbeitung kirchlicher Themen in den letzten zwei Wochen}

In den Antworten auf die Frage "Wie oft haben Sie in den letzten zwei Wochen Artikel über kirchliche Themen geschrieben?" zeigte sich ein dreigeteiltes Bild. Die große Mehrheit der befragten Redakteure (65,5\%) schrieb kein einziges Mal in den vergangenen 14 Tagen über kirchliches Leben. Eine kleinere Gruppe $(24,1 \%)$ berichtete ein- bis dreimal in diesem Zeitraum über Kirche, die kleinste Gruppe $(10,3 \%)$ berichtete sehr häufig (sechs- bis achtmal im Zeitraum) über Kirche.

Die Häufigkeit der bearbeiteten Kirchenthemen korreliert dabei signifikant $(p<0,05)$ mit den Antworten $z \mathfrak{u}$ den Fragen nach der persönlichen Religiosität sowie der Kirchenverbundenheit und -vertrautheit. Das heißt: Je stärker ein Redakteur mit der Kirche vertraut und verbunden sowie religiös ist, desto häufiger schreibt er über Religion und Kirche.

\subsection{Verbesserungsmöglichkeiten für die Kommunikation zwischen Kirche und Medien aus der Perspektive der befragten Redakteure}

Auf die offene Frage: „Welche Verbesserungsmöglichkeiten sehen Sie für die Zusammenarbeit zwischen Kirche und Medien?" antworteten 15 Redakteure und damit mehr als die Hälfte der Stichprobe mit relativ ausführlichen Hinweisen. Diese Resonanz kann ein Hinweis auf zweierlei sein: Einmal, dass die befragten Redakteure dem Dialog mit Kirche keineswegs gleichgültig gegenüberstehen und dass sie an einer Verbesserung in der Kommunikation durchaus - zunächst ein berufsmäßiges - Interesse haben. Und zweitens, dass in der Kommunikation mit der Kirche Schwachpunkte gesehen werden, die sowohl technischer wie menschlicher Natur sind. Diese Vorschläge sollen im Folgenden aufgeführt und zusammengefasst werden, ohne dass sie quantifiziert werden können:

Pressemitteilungen über kirchliche Aktionen und Veranstaltungen sollten regelmäßig und vor allem rechtzeitig im Vorfeld des Ereignisses die Redaktionen erreichen. Die Redakteure haben jedoch keineswegs nur Interesse an Terminen, sondern auch an „innerkirchlichen Entwicklungen“, darüber würden sie gern mehr Informationen erhalten. Für einen besseren Informationsaustausch mit der Kirche sei sowohl professionell wie menschlich ein "kurzer Draht" zwischen kirchlichen Mitarbeitern und Redakteuren wünschenswert: per Telefon oder mittels Internet und E-Mail. Hier müssten „Hemmschwellen“ auf kirchlicher Seite gesenkt werden, finden einige Journalisten. Zudem sei mehr Eigeninitiative von Seiten der Kirclfe bei der Versorgung der Medien mit kirchlichen Informationen wünschenswert, und dafür wäre ein lokaler kirchlicher „Medienbeauftragter" hilfreich. 
In der emotionalen Dimension der Kommunikation zwischen Kirche und Redakteuren sehen einige Redakteure den „Abbau von Berührungsängsten auf beiden Seiten (als) dringend notwendig“ an. Von Seiten der kirchlichen Mitarbeiter wünschen sich Redakteure mehr Offenheit sowie mehr Transparenz - und dass Journalisten mehr als „Partner" gesehen werden statt als „bei Bedarf verfügbares Mittel zum Zweck“.

\subsection{Die Einstellung kirchlicher Mitarbeiter gegenüber Journalisten aus der Perspektive der befragten Redakteure}

Bei den Antworten auf die Frage „Wie empfinden Sie die Einstellung kirchlicher Mitarbeiter gegenüber Journalisten?" ergab sich ein dreigeteiltes Bild dreier signifikant unterschiedener Gruppen: Bezieht man den großen Anteil ungültiger bzw. nicht gegebener Antworten (20,7\%) nicht mit ein, so wählte eine Mehrheit von 52,2\% zur Bezeichnung der Einstellung kirchlicher Mitarbeiter gegenüber den Journalisten die Formel „freundlich-entgegenkommend“. Immerhin 30,4\% wählten „distanziert-kritisch“ und $17,4 \%$,neutral-gleichgültig“. „Ablehnend-feindlich“ fand keine $\mathrm{Zu}$ stimmung.

\subsection{Die Bekanntheit des Bischofs der evangelisch-lutherischen Landes- kirche Sachsens}

Auf die Frage „Kennen Sie den Bischof der evangelisch-lutherischen Landeskirche Sachsens?" antworteten 48,3\% der Befragten mit "nein“, ebenfalls $48,3 \%$ mit ,ja“, eine Antwort war ungültig.

Auch wenn man bei dieser Fragestellung eine gewisse Unschärfe in Rechnung stellen muss (Was bedeutet "kennen"?) und auch beachten sollte, dass die schlesische Lausitz sowie das Gebiet um Delitzsch, Eilenburg und Torgau nicht zur sächsischen Landeskirche gehören, so zeigt dieses Ergebnis doch zumindest eine Tendenz, dass der sächsische Landesbischof unter einem erheblichen Teil der von Berufs wegen gut informierten Redakteure nicht bekannt ist. ${ }^{12}$

\section{Zusammenfassung und Ausblick}

Der Blick der befragten ostdeutschen Redakteure auf die evangelische Kirche ist ein Blick aus der Distanz. Die Botschaft der Kirche hat - wie Religion überhaupt - für die meisten von ihnen keine existentielle Bedeutung. Daraus jedoch zu folgern, dass kirchliches Leben für die Redakteu-

12 Die Erhebung fand im Jahre 2003 statt und damit vor dem Wechsel im Amt des sächsischen Landesbischofs. 
re keine journalistische Relevanz habe, wäre zu voreilig. Die vorliegende Untersuchung zeigt vielmehr, dass eine große Breite an kirchlichen Themen von den befragten Redakteuren durchaus relativ hohe Priorität zugemessen bekommt. Und sie zeigt auch, dass eine große Gruppe unter den befragten Redakteuren die gesellschaftlichen Leistungen der Kirche für Moral, Kultur und für sozial Schwache anerkennend wahrnimmt. Daraus ließe sich die These aufstellen: Gerade deren geringe private Religiosität lässt die befragten ostdeutschen Redakteure die Relevanz von "Religion" und „Kirche“ nicht zuerst im Privaten suchen, sondern im öffentlichen Raum der Gesamtgesellschaft.

Als Konsequenz für kirchliche Öffentlichkeitsarbeit in Ostdeutschland hieße dies: Nicht christliche Lebensberatung und christlicher Lifestyle, nicht modisch, stromlinienförmig und harmlos daherkommende Medienangebote sind hier gefragt, sondern der freundlich-positive bis neutralskeptische Blick der befragten ostdeutschen Journalisten richtet sich hauptsächlich auf das kulturelle, ethische, kritisch-politische und vor allem soziale Engagement der Kirche in der Gesellschaft und blendet auch spirituelle Lebensäußerungen der Kirche nicht grundsätzlich aus. Dies könnte sich als Chance für die mediale Präsenz der Kirche erweisen, das Evangelium in seiner ihm untrennbar eigenen sozialen und politischen Dimension zur Sprache zu bringen, so dass man darin eine Art der Verkündigung sehen kann. Gerade in Ostdeutschland, wo gegenüber der institutionellen Hülle der Kirche weitgehend Desinteresse herrscht, könnte eine solche „kommunikative Diakonie“ (Peter Düsterfeld) bei den mehrheitlich areligiösen und kirchenfernen Journalisten und deren $\mathrm{Pu}$ blikum Gehör finden. Denn nicht zuletzt wegen der zwei großen deutschen Diktaturen ist man in Ostdeutschland offenbar skeptisch gegenüber aller ideologischen Phraseologie, auch der religiösen. Umso geschärfter ist womöglich der ostdeutsche Blick darauf, ob die Taten einhalten, was die Worte versprechen. Eine Kirche in der Öffentlichkeit, die das Evangelium in seinen sozialen und politischen Konsequenzen mutig und kommunikativ lebt, könnte eine Quelle sein für das „rumorende Gerücht" (Ottmar Fuchs), das sich schon um Jesus von Nazareth ausbreitete. Die in dieser Untersuchung befragten Redakteure würden ein solches „rumorendes Gerücht" nicht überhören.

Gleichzeitig aber hat die Untersuchung Hinweise auf Mängel und Barrieren in der Kommunikation zwischen Kirche und Medien im sächsischen Untersuchungsfeld aufgezeigt. Wenn man sich der These anschließt, dass Verkündigung auch über die Kanäle der Massenmedien möglich ist, dann̂ muss die Verbesserung der Kommunikation zwischen Kirchenvertretern und Journalisten eine zentrale Aufgabe der Kirche sein. Die Untersuchung ergab Hinweise darauf, dass wahrscheinlich aus 
Erfahrungen während der SED-Herrschaft herrührende Defizite in der zwischenmenschlichen Kommunikation zwischen Kirchenvertretern und Journalisten wichtige Gründe für die mangelhafte Präsẻnz der Kirche in den Medien sein könnten. Ein Wandel von Einstellungen und mentaler Distanz jedoch ist nur schwer herbeizuführen, aber nicht unmöglich.

Dass dieser mentale Graben zwischen Kirche und Medien überbrückbar ist, dafür haben die befragten Redakteure selbst Ansätze einer Lösung mitgeliefert. Ein Dialog zwischen journalistischen und kirchlichen Mitarbeitern, notierten einige Befragte, würde gegenseitiges Verständnis über die jeweiligen Verstehensmuster und Erfahrungen, vor allem aber über die Möglichkeiten und Grenzen der Arbeit des Gegenübers wie z.B. über den journalistischen Zeitdruck und den Zwang zur Verkürzung entwickeln helfen. Genauso wären kirchliche Workshops, Weiterbildungsangebote, Hintergrundgespräche mit Experten etwa zu ethischen und sozialen Themen für Journalisten denkbar, denn gerade in diesen - journalistisch relevanten - Bereichen fehlt vielen Redakteuren oft ein tiefergehendes Wissen; gleichzeitig sehen sie in der Kirche eine dafür kompetente Institution. Auf diese Weise könnte die Kirche zur Partnerin der Medienarbeiter werden, und - quasi nebenbei - sie mit Kirche vertraut machen. Denn die Studie erbrachte Hinweise auf diesen Zusammenhang: Je vertrauter ein Redakteur mit Kirche ist, desto öfter schreibt er über sie.

Dafür aber ist nach Ansicht der befragten Redakteure mehr kirchliches Engagement, mehr Offenheit und Partnerschaft seitens der Kirche in der Zusammenarbeit mit den Medien nötig. Die Respondenten wünschten sich mehr und vor allem regelmäßige Kontakte sowie kirchliche Ansprechpartner, die die Medien mit interessanten Informationen aus den Gemeinden rechtzeitig versorgen - es ist dies das Modell eines gemeindeübergreifenden regionalen „Medienkoordinators“. Ein solcher „Medienkoordinator" hätte auch die Aufgabe, die Gemeinden immer wieder an ihren Auftrag zur Öffentlichkeitsarbeit zu erinnern, sie dafür zu qualifizieren - und zu ermutigen. Eine solche Ermutigung, das hat diese empirische Studie gezeigt, wäre nicht das kirchliche Pfeifen im dunklen Wald eines areligiösen Ostdeutschlands: Eine solche Ermutigung dürfte nämlich darauf bauen, dass viele ostdeutsche Journalisten - obwohl selbst weder religiös noch Kirchenmitglied - die sozialen, politischen und kulturellen Implikationen des gelebten Evangeliums mit Interesse und Aufgeschlossenheit verfolgen.

Schließlich noch ein methodischer Ausblick: Um eine größere Genauigkeit, Verlässlichkeit und Repräsentativität der Daten zu erreichen, müss- te für eine weiterführende Studie die Stichprobe vergrößert, der Fragebogen anhand der Ergebnisse der vorliegenden Untersuchung ver- 
bessert, demographische Daten zur Grundgesamtheit gewonnen sowie Re-Tests durchgeführt werden, um die Stabilität des Meinungsbildes zu prüfen. Auch wäre die Befragung einer westdeutschen Vergleichsgruppe denkbar, damit die Ergebnisse besser eingeordnet werden können. Um die Handlungsrelevanz der von den Befragten gegebenen Auskünfte genauer zu prüfen, wäre eine Inhaltsanalyse der Medien, in denen die Befragten arbeiten, eine weiterführende Methode. Für eine solche Studie würde sich die interdisziplinäre Zusammenarbeit der Praktischen Theologie und der Religionssoziologie mit Kommunikationswissenschaftlern anbieten. Eine solche Kooperation würde sich lohnen angesichts der Bedeutung der „Meinungsmacher" für die Gesellschaft und deren Einstellungen. 